\section{P053 LNCRNA HOTAIR PROMOTES PROLIFERATION AND INVASION OF FIBROBLAST-LIKE SYNOVIOCYTES AS MICRORNA SPONGING IN RA PATIENTS}

X Bi, YF Pan*, XQ Luo, ML Wang, YX Chen. Department of Rheumatology, The Third Affiliated Hospital of Sun Yat-Sen University, Guangzhou, China

10.1136/annrheumdis-2018-EWRR2019.45

Career situation of first and presenting author: Student for a master or a $\mathrm{PhD}$.

Introduction Long non-coding RNAs (lncRNAs) have drawn increasing attention because of the pivotal roles which they play in various types of autoimmune diseases, including rheumatoid arthritis (RA). LncRNA HOTAIR is a crucial lncRNA function as an oncogene in multiple cancers. Fibroblast-like synoviocytes (FLSs), a prominent component of hyperplastic synovial pannus tissue, are critical to synovial aggression and joint destruction in RA. However, the functions of lncRNA and the potential mechanisms remain to be further elucidated in FLSs of RA patients.

Objectives Our present study aimed to investigate the expression and roles of IncRNA HOTAIR in RA-FLSs and explore its possible mechanism.

Methods FLSs were cultured from synovial tissues of join. LncRNA and mircoRNA expression profiles in FLSs were screened by microarrays, and then we validated the results by Real-time Quantitative polymerase chain reaction (qRTPCR). Small interfering RNA (siRNA) was then used to knock down the expression of HOTAIR in order to determine its role in RA FLSs. Cell viability was evaluated using the CCK-8 assay and flow cytometry. Cell invasion was analyzed by transwell chamber methodology. Bioinformatics analysis were performed to predict the possible competitive endogenous RNA (ceRNA) mechanisms via miRanda, PITA, RNAhybrid, as well as KEGG and Gene Ontology(GO) analysis.

Results Both microarray analysis and qRT-PCR showed the expressions of IncRNA HOTAIR were up-regulated in RA FLSs compared with healthy controls (HCs). Transfection of HOTAIR-siRNA significantly decreased the expression of lncRNA HOTAIR in RA FLSs. HOTAIR knockdown largely inhibited cell proliferation and invasion of RA FLSs. Furthermore, the bioinformatics analysis predicted that some of microRNAs and mRNAs may be the downstream molecules of IncRNA HOTAIR. Considering the mircoRNA expression profiles detected by microarrays and the results from qRT-PCR, we designated miR-138 and miR-17-5 $\mathrm{p}$ as potential ceRNAs which IncRNA HOTAIR could directly bind to. In addition, the expressions of miR-138 and miR17-5 p were markedly downregulated in RA FLSs, whereas the knockdown of IncRNA HOTAIR upregulated the expressions compared with the negative control group (NCsiRNA).

Conclusions Our study illuminated that elevated lncRNA HOTAIR expression promoted the proliferation and invasion of RA FLSs. Meanwhile, it may function as a novel microRNAs sponging agent and regulate RA FLSs pathological behaviors via miR-138 or miR-17-5 $\mathrm{p}$ associated
ceRNA network. In summary, the regulation of lncRNA HOTAIR may be a promising therapeutic strategy for RA in the future.

Acknowledgements This work was supported by grants provided from Province Natural Science Fund of Guangdong, China (No.2014A030313080) and National Natural Science Foundation of China (No.81771750).

Disclosure of Interest None declared

\section{P054 IRF-1 IS CRITICAL FOR THE TNF-DRIVEN INTERFERON RESPONSE IN RHEUMATOID FIBROBLAST-LIKE SYNOVIOCYTES}

${ }^{1} \mathrm{M}$ Bonelli*, ${ }^{1} \mathrm{~K}$ Dalwigk, 'A Platzer, ${ }^{1} \mathrm{I}$ Calvo, ${ }^{1} \mathrm{~S}$ Hayer, ${ }^{1} \mathrm{~B}$ Niederreiter, ${ }^{2} \mathrm{~J}$ Holinka, ${ }^{2} \mathrm{~F}$ Sevelda, ${ }^{3} \mathrm{~T}$ Pap, ${ }^{1} \mathrm{G}$ Steiner, ${ }^{4} \mathrm{G}$ Superti-Furga, ${ }^{1} \mathrm{~J}$ Smolen, ${ }^{1} \mathrm{H}$ Kiener, ${ }^{1} \mathrm{~T}$ Karonitsch. ${ }^{1}$ Division of Rheumatology; ${ }^{2}$ Department of Orthopedics, Medical University of Vienna, Vienna, Austria; ${ }^{3}$ University Hospital Muenster, Muenster, Germany; ${ }^{4}$ CeMM Research Center for Molecular Medicine of the Austrian Academy of Sciences, Vienna, Austria

\subsection{6/annrheumdis-2018-EWRR2019.46}

Career situation of first and presenting author Young investigator.

Introduction Rheumatoid arthritis (RA) is an autoimmune disease, which is characterized by persistent, synovial inflammation. Major drivers of synovial inflammation are cytokines and chemokines. Among them TNF activates fibroblast-like synoviocytes (FLS), which leads to the production of inflammatory mediators. The pathways and transcription factors that determine the inflammatory response in FLS are largely unexplored. Here, we investigated the potential contribution of the transcription-factor IRF1 to the inflammatory gene expression in FLS.

Methods Expression of IRF1 in synovial tissues was assessed by immunohistochemistry (IHC). RA-FLS were isolated according to established protocols and cultured using 2-D or 3-D culture techniques. IRF1 expression in response to TNF was determined by western blots, qPCR or IHC. FLS were also stimulated with TNF in the presence or absence of IRF1 siRNA pools. Global changes of mRNA expression were assessed by RNA-sequencing. Janus kinase activity was blocked by baricitinib or tofacitinib.

Results Our data reveal that TNF regulates the expression of IRF1 in human FLS as well as in the huTNFtg mouse model of arthritis. Transcriptomic analyses of IRF-1-deficient TNF-stimulated FLS define the interferon (IFN) pathway as a major target of IRF-1. Further experiments show that TNF-induced IRF-1 expression promotes the expression of IFN- $\beta$, which leads to an activation of the JAKSTAT pathway. Blockade of the JAK-STAT pathway, by the Janus kinase inhibitors baricitinib or tofacitinib, reduces the expression of IFN-regulated genes (IRGs), such as CXCL9, CXCL10, CXCL11 and TNFSF13B, in TNF-activated FLS.

Conclusions Our data reveal that IRF1 is crucial for the IFNresponse of FLS and support the idea that IRF1 plays a critical role for the development of synovial inflammation.

Disclosure of Interest None declared. 